\title{
Some Accepting Powers of Bottom-Up Pyramid Cellular Acceptors with $\boldsymbol{n}$-dimensional Layers
}

\author{
Makoto Sakamoto*, Makoto Nagatomo, Tatsuma Kurogi, Satoshi Ikeda, \\ Masahiro Yokomichi and Hiroshi Furutani \\ Faculty of Engineering, University of Miyazaki, Miyazaki 889-2192, Japan
}

Takao Ito

Institute of Engineering, Hiroshima University, Higashi-Hiroshima, Hiroshima 739-8527, Japan

Yasuo Uchida

Department of Business Administration, Ube National College of Technology,Ube, Yamaguchi 755-8555, Japan

Tsunehiro Yoshinaga

Department of Computer Science and Electronic Engineering, Tokuyama college of Technology, Shunan, Yamaguchi 745-8585, Japan

*Corresponding author, E-mail: sakamoto@cs.miyazaki-u.ac.jp

Tel: +81-985-58-7392, Fax: +81-985-58-7392

\begin{abstract}
In theoretical computer science, the Turing machine was introduced as a simple mathematical model of computers in 1936, and has played a number of important roles in understanding and exploiting basic concepts and mechanisms in computing and information processing. After that, the development of the processing of pictorial information by computer was rapid in those days. Therefore, the problem of computational complexity was also arisen in the two-dimensional information processing. M.Blum and C.Hewitt first proposed two-dimensional automata as a computational model of two-dimensional pattern processing in 1967[1]. Since then, many researchers in this field have been investigating many properties of two- or three-dimensional automata. In 1997, C.R.Dyer and A.Rosenfeld introduced an acceptor on a two-dimensional pattern (or tape), called the pyramid cellular acceptor, and demonstrated that many useful recognition tasks are executed by pyramid cellular acceptors in time proportional to the logarithm of the diameter of the input. They also introduced a bottom-up pyramid cellular acceptor which is a restricted version of the pyramid cellular acceptor, and proposed some interesting open problems about bottom-up pyramid cellular acceptors. On the other hand, we think that the study of $n$-dimensional automata has been mean- ingful as the computational model of $n$-dimensional information processing[9]. In this paper, we investigate about bottom-up pyramid cellular accptors with n-dimensional layers, and show their some accepting powers.
\end{abstract}

Keywords: cellular automaton, diameter, finite automaton, $n$-dimension, parallelism, pattern recognition, real time.

\section{Introduction}

In 1967, M.Blum and C.Hewitt first proposed twodimensional automata as a computational model of two-dimensional pattern processing, and investigated their pattern recognition abilities [1]. Since then, many researchers in this field have been investigating a lot of properties about automata on a two- or three-dimensional tape. In [2], C.R.Dyer and A.Rosenfeld introduced an acceptor on a two-dimensional pattern (or tape), called the pyramid cellular acceptor, and demonstrated that many useful recognition tasks are executed by the pyramid cellular acceptors in time proportional to logarithm of the diameter of the input. They also introduced a bottom-up pyramid cellular acceptor, which 
is a restricted version of the pyramid cellular acceptor, and proposed some interesting open problems about it. On the other hand, the question of whether processing $n$-dimensional digital patterns is much difficult than (n-1)-dimensional ones is of great interest from the theoretical and practical standpoints. Thus, the study of n-dimensional automata as the computational model of $n$-dimensional pattern processing has been meaningful. From this point of view, we are interested in n-dimensional automata.

In this paper, we study about bottom-up pyramid cellular acceptors with $n$-dimensional layers, and deal with the following problems (which is one of the open problems) : Does the class of sets accepted by deterministic bottom-up pyramid cellular acceptors with $n$-dimensional layers include the class of sets accepted by deterministic $n$-dimensional finite automata [3-7]? This paper shows that the class of sets accepted by $n$-dimensional finite automata is incomparable with the class of sets accepted by deterministic bottom-up pyramid cellular acceptors which operate in time of order lower than the diameter of the input.

\section{Definition}

Let $\Sigma$ be a finite set of symbols. An $n$-dimensional tape over $\Sigma$ is an $n$-dimensional array of elements of $\Sigma$. The set of all the $n$-dimensional tapes over $\Sigma$ is denoted by $\Sigma^{(n)}$. Given a tape $x \in \Sigma^{(n)}$, for each $j(1 \leq j \leq n)$, we let $l_{j}(x)$ be the length of $x$ along the $j$ th axis. The set of all $x \in \Sigma^{(n)}$ with $l_{1}(x)$ $=n_{1}, l_{2}(x)=n_{2}, \ldots, l_{n}(x)=n_{n}$ is denoted by $\Sigma^{\left(n_{1}, n_{2}, \ldots, n_{n}\right)}$. When $1 \leq i_{j} \leq l_{j}(x)$ for each $j(1 \leq j \leq n)$, let $x\left(i_{1}, i_{2}, \ldots, i_{n}\right)$, denote the symbol in $x$ with coordinates $\left(i_{1}, i_{2}, \ldots, i_{n}\right)$. Furthermore, we define $x\left[\left(i_{1}, i_{2}, \ldots, i_{n}\right),\left(i_{1}{ }^{\prime}, i_{2}, \ldots, i_{n}{ }^{\prime}\right)\right]$, when $i \leq i_{j} \leq i_{j}{ }^{\prime} \leq$ $l_{j}(x)$ for each integer $j(1 \leq j \leq n)$, as the $n$-dimensional input tape $y$ satisfying the following (i) and (ii) : (i) for each $j$ ( $1 \leq j$ $\leq n), l_{j}(y)=i_{j}{ }^{\prime}-i_{j}+1$; (ii) for each $r_{1}, r_{2}, \ldots, r_{n}\left(1 \leq r_{1} \leq l_{1}(y), 1\right.$ $\left.\leq r_{2} \leq l_{2}(y), \ldots, 1 \leq r_{n} \leq l_{n}(y)\right), y\left(r_{1}, r_{2}, \ldots, r_{n}\right)=x\left(r_{1}+i_{1}-1\right.$, $\left.r_{2}+i_{2}-1, \ldots, r_{n}+i_{n}-1\right)$.

We next give some basic concepts about bottom-up pyramid cellular acceptors with n-dimensional layers [7]. A bottom-up pyramid cellular acceptor with n-dimensional layers $(n-U P C A)$ is a pyramidal stack of $n$-dimensional arrays of cells in which the bottom $n$-dimensional layer has size $2^{t} \times 2^{t}$ $\times \cdots \times 2^{t}(t \geq 0)$, the next lowest $2^{t-1} \times 2^{t-1} \times \cdots \times 2^{t-1}$, and so forth, the $(t+1)$ st $n$-dimensional layer consisting of a single cell, called the root. Each cell is defined as an identical finite-state machine, $M=\left(Q_{N}, Q_{T}, \delta, A\right)$, where $Q_{N}$ is a nonempty, finite set of states, $Q_{T} \subseteq Q_{N}$ is a finite set of input states, $A \subseteq Q_{N}$ is the set of accepting states, and $\delta: Q_{N}^{2^{n}+1}$ $\rightarrow Q_{N}$ is the state transition function, mapping the current states of $M$ and its $2^{n}$ son cells in a $2 \times 2 \times \cdots \times 2$ block on the $n$-dimensional layer below into $M$ 's next state. For example, the definition in the case of four-dimensional case as follows. Let $c$ be some cell on the $(i+1)$ st $n$ - dimensional layer, and let $c$ (UNWP), c (UNWF), c (USWP), c (USWF), c (USEP), c (USEF), c (UNEP), c (UNEF), c (DNWP), c (DNWF), c (DSWP), c (DSWF), c (DSEP), c (DSEF), c (DNEP), and c (DNEF) be sixteen son cells (on the $i$ th $n$-dimensional layer) of $c$, where $c(U N W F)$ is $c$ 's upper northwest son in the most future direction, $c(D N W P)$ is c's lower northwest son in the most past direction, etc. For example, if the coordinates of $c$ on the $(i+1)$ st layer is $(1,1,1,1)\left(\left(2^{t}, 2^{t}, 2^{t}, 2^{t}\right)\right)$, the coordinates of sixteen son cells of $c$ on the ith layer $c$ (UNWP), c (UNWF), c (USWP), c (USWF), c (USEP), c (USEF), c (UNEP), c (UNEF), c (DNWP), c (DNWF), c (DSWP), c (DSWF), c (DSEP), c (DSEF), c (DNEP), and c (DNEF) are (1, $1,1,1),(1,1,1,2),(1,2,1,1),(1,2,1,2),(1,2,2,1),(1,2,2$, 2), (1, 1, 2, 1), (1, 1, 2, 2), (2, 1, 1, 1), (2, 1, 1, 2), (2, 2, 1, 1), (2, 2, 1, 2), (2, 2, 2, 1), (2, 2, 2, 2), (2, 1, 2, 1), (2, 1, 2, 2), ((2t $\left.-1,2^{t}-1,2^{t}-1,2^{t}-1\right),\left(2^{t}-1,2^{t}-1,2^{t}-1,2^{t}\right),\left(2^{t}-1,2^{t}\right.$, $\left.2^{t}-1,2^{t}-1\right),\left(2^{t}-1,2^{t}, 2^{t}-1,2^{t}\right),\left(2^{t}-1,2^{t}, 2^{t}, 2^{t}-1\right),\left(2^{t}-\right.$ $\left.1,2^{t}, 2^{t}, 2^{t}\right),\left(2^{t}-1,2^{t}-1,2^{t}, 2^{t}-1\right),\left(2^{t}-1,2^{t}-1,2^{t}, 2^{t}\right),\left(2^{t}\right.$, $\left.2^{t}-1,2^{t}-1,2^{t}-1\right),\left(2^{t}, 2^{t}-1,2^{t}-1,2^{t}\right),\left(2^{t}, 2^{t}, 2^{t}-1,2^{t}-1\right)$, $\left(2^{t}, 2^{t}, 2^{t}-1,2^{t}\right),\left(2^{t}, 2^{t}, 2^{t}, 2^{t}-1\right),\left(2^{t}, 2^{t}, 2^{t}, 2^{t}\right),\left(2^{t}, 2^{t}-1,2^{t,}\right.$ $\left.\left.2^{t}-1\right),\left(2^{t}, 2^{t}-1,2^{t}, 2^{t}\right)\right)$, respectively. Then $q_{c}(t+1)=\delta\left(q_{c}(t)\right.$, $q_{c(\text { UNWP })}(t), q_{c(U N W F)}(t), q_{c(\text { USWP })}(t), q_{c(\text { USWF })}(t), q_{c(\text { USEP })}(t)$, $q_{c(U S E F)}(t), q_{c(\text { UNEP })}(t), q_{c(\text { UNEF })}(t), q_{c(D N W P)}(t), q_{c(D N W F)}(t)$, $q_{c(D S W P)}(t), q_{c(D S W F)}(t), q_{c(D S E P)}(t), q_{c(D S E F)}(t), q_{c(D N E P)}(t)$, $\left.q_{c(D N E F)}(t)\right)$, where for example $q_{c}(t)$ means the state of $c$ at time $t$. At time $t=0$, the input tape $x \in Q_{T}^{(4)}\left[l_{1}(x)=l_{2}(x)=\right.$ $\left.l_{3}(x)=l_{4}(x)=2^{t}, \mathrm{t} \geq 0\right]$ is stored as the initial states of the bottom $n$-dimensional layer, henceforth called the base, in such a way that $x\left(i_{1}, i_{2}, i_{3}, i_{4}\right)$ is stored at the cell of the $i_{1}$ th row and the $i_{2}$ th column on the $i_{3}$ th plane of the $i_{4}$ th n-dimensional rectangular array, and the other cells are initialized to a quiescent state $q_{s}\left(\in Q_{N}-Q_{T}-A\right)$. As usual, we let $\delta\left(q_{s}, q_{s}, q_{s}, q_{s}, q_{s}, q_{s}, q_{s}, q_{s}, q_{s}, q_{s}, q_{s}, q_{s}, q_{s}, q_{s}, q_{s}, q_{s}, q_{s}\right)=$ $q_{s}$. The input is accepted if and only if the root cell ever enters an accepting state. This 4-UPCA is called deterministic. A nondeterministic bottom-up pyramid cellular acceptor is defined as a 4-UPCA using $\delta: Q_{N}^{17} \rightarrow 2^{Q_{N}}$ instead of the state transition function of the deterministic 4-UPCA. Below, we denote a deterministic n-UPCA by $n$-DUPCA, and a nondeterministic $n$-UPCA by $n$-NUPCA. An $n$-DUPCA (or $n$-NUPCA) operates in time $T(n)$ if for every $n$-dimensional 
tape of size $2^{t} \times 2^{t} \times 2^{t} \times 2^{t}(t \geq 0)$ it accepts the $n$-dimensional tape, then there is an accepting computation which uses no more than time $T(t)$. By $n$-DUPCA $(T(t))[n-N U P C A(T(t))]$ we denote a $T(t)$ time-bounded n-DUPCA [n-NUPCA] which operates in time $T(t)$.

We next introduce an $n$-dimensional finite automaton [8]. An $n$-dimensional finite automaton $(n-F A)$ is an $n$-dimensional Turing machine with no workspace. An $n-F A M$ has a read-only $n$-dimensional tape with boundary symbols \#'s, finite control, and an input head. For example, we explain the definition in the case of four-dimensional case as follows. The input head can move in eight direction - east, west, south, north, up, down, future, or past - unless it falls off the input tape. Formally, $M$ is defined by the 5-tuple $M=(K, \Sigma \cup\{\#\}, \delta$, $q_{0}, F$ ), where $K$ is a finite set of states, $\Sigma$ is a finite set of input symbols, \# is the boundary symbol (not in $\Sigma$ ), $\delta: K \times$ $(\Sigma \cup\{\#\}) \rightarrow 2^{K \times\{E, W, S, N, U, D, F, P, H\}}$ is the state transition function, where $E, W, S, N, U, D, F, P$, and $H$ represent the move directions of the input head - east, west, south, north, up, down, future, past, and no move, respectively, $q_{0} \in K$ is the initial state, and $F \subseteq K$ is the set of accepting states. The action of $M$ is similar to that of the one-dimensional (or two-dimensional) finite automaton [4], except that the input head of $M$ can move in eight directions. That is, when an input tape $x \in \Sigma^{(4)}$ with boundary symbols is presented to $M, M$ starts in its initial state $q_{0}$ with the input head on $x(1,1,1,1)$, and determines the next state of the finite control and the move direction of the input head, depending on the present state of the finite control and the symbol read by the input head. We say that $M$ accepts the tape $x$ if it eventually enters an accepting state. We denote a deterministic $n$ - $F A$ [nondeterministic $n-F A$ ] by $n-D F A$ [ $n-N F A]$.

We let each sidelength of each input tape of $n$-dimensional automata, throughout this paper, be equivalent. We denote the set of all $n$-dimensional tapes accepted by $M$ by $T(M)$. Define $£[n-D U P C A]=\{T \mid T(M)$ is accepted by some $n$-DUPCA $M\}$. $£[n-N U P C A], £[n-D F A]$, etc. are defined similarly.

Finally, we give definition of diameter. For example, we explain the definition in the case of four-dimension. Given a subset $\mathrm{S}$ of a tape $x \in \Sigma^{(4)}$, we can define its extent in a given direction $\theta$ as the length of its projection on a plane in that direction. Here the length of a projection is the distance between its farthest apart nonzero values. Thus the extent of $\mathrm{S}$ is the distance between a pair of parallel planes perpendicular to $\theta$ that just bracket $\mathrm{S}$. The diameter of $\mathrm{S}$ is defined as its extent in any direction.

\section{Results}

In this section, we show that the class of sets accepted by $n$-DFA's is incomparable with the class of sets accepted by $n$-DUPCA's which operate in time of order lower than the diameter of the input. It has often been noticed that we can easily get several properties of $n$-dimensional automata by directly applying the results of (n-1)-dimensional case, if each sidelength of each $n$-dimensional input tape of these automata is not equivalent. So we let each sidelength of each input tape, throughout this paper, be equivalent in order to increase the theoretical interest.

Lemma 3.1. Let $T_{1}=\left\{x \in\{0,1\}^{(n)} \mid \exists t(t \geq 1)\left[\ell_{1}(x)=\right.\right.$ $\left.\ell_{2}(x)=\cdots=\ell_{n}(x)=2^{t}\right]$ and $\left.x\left(2^{t-1}, 2^{t-1}, \ldots, 2^{t-1}\right)=1\right\}$. Then,

(1) $T_{1}(x) \notin £[n-D F A]$, and

(2) $T_{1}(x) \in £[n-D U P C A(t)]$.

Proof : The Proof of (1) is similar to that of Theorem 3 in [7]. On the other hand, by using the same technique as in the proof of Lemma 1 in [6], we can get Part (2) of the lemma.

Lemma 3.2. Let $T_{2}=\left\{x \in\{0,1\}^{(n)} \mid \exists t(t \geq 1)\left[\ell_{1}(x)=\ell_{2}(x)\right.\right.$ $\left.=\cdots=\ell_{n}(x)=2^{t}\right]$ and $x\left[(1,1, \ldots, 1),\left(2^{t}, 2^{t}, \ldots, 1\right)\right]=x[(1$, $\left.\left.\left.1, \ldots, 2^{t}\right),\left(2^{t}, 2^{t}, \ldots, 2^{t}\right)\right]\right\}$. Let $T(t)$ be a time function such that $\lim _{t \rightarrow \infty}\left[T(t) / 2^{2^{t}}\right]=0$. Then,

(1) $T_{2} \in £[n-D F A]$, and

(2) $T_{2} \notin £[n-D U P C A(T(t))]$.

Proof : It is obvious that there is an $n-D F A$ accepting $T_{2}$, and so (1) of the lemma holds. Below, we prove (2). Suppose that there is an $n$-DUPCA $B$ which accepts $T_{2}$ and operates in time $T(t)$, and that each cell of $B$ has $k$ states. For each $t \geq 2$, let

$$
\begin{aligned}
& W(t)=\left\{x \in\{0,1\}^{(n)} \mid \ell_{1}(x)=\ell_{2}(x)=\cdots=\ell_{n}(x)=2^{t}\right\}, \text { and } \\
& W^{\prime}(n)=\left\{x \in\{0,1\}^{(n)} \mid \ell_{1}(x)=\ell_{2}(x)=\cdots=\ell_{n}(x)=2^{t-1}\right. \\
& \& x\left[(1,1, \ldots, 1),\left(2^{t-1}, 2^{t-1}, \ldots, 1\right)\right] \in\{0,1\}^{(n)} \\
& \left.\& x\left[(1,1, \ldots, 2),\left(2^{t-1}, 2^{t-1}, \ldots, 2^{t-1}\right)\right] \in\{0\}^{(n)}\right\} .
\end{aligned}
$$

We consider the cases when the tapes in $W(t)$ are presented to $B$. Let $c$ be the cell which is situated at the first row, the first column, the first plane, $\ldots$, and the first (n-1)-dimensional array in the th layer (i.e., the layer just below the root cell). For each $x$ in $W(t)$ such that $x\left[(1,1, \ldots, 1),\left(2^{t-1}, 2^{t-1}, \ldots\right.\right.$, $\left.\left.2^{t-1}\right)\right] \in W^{\prime}(t)$, and for each $r \geq 1$, let $q_{r}(x)$ be the state of $c$ at time $r$ when $x$ is presented to $B$. Then the following proposition must hold. 
Proposition 3.1. Let $x, y$ be two different tapes in $W(t)$ such that both $x\left[(1,1, \ldots, 1),\left(2^{t-1}, 2^{t-1}, \ldots, 2^{t-1}\right)\right]$ and $y[(1,1, \ldots$, $\left.1),\left(2^{t-1}, 2^{t-1}, \ldots, 2^{t-1}\right)\right]$ are in $W^{\prime}(t)$ and $x\left[(1,1, \ldots, 1),\left(2^{t-1}\right.\right.$, $\left.\left.2^{t-1}, \ldots, 2^{t-1}\right)\right] \neq y\left[(1,1, \ldots, 1),\left(2^{t-1}, 2^{t-1}, \ldots, 2^{t-1}\right)\right]$. Then, $\left(q_{1}(x), q_{2}(x), \ldots, q_{T(t)}(x)\right)=\left(q_{1}(y), q_{2}(y), \ldots, q_{T(t)}(y)\right)$.

[Proof : For suppose that $\left(q_{1}(x), q_{2}(x), \ldots, q_{T(t)}(x)\right)=\left(q_{1}(y)\right.$, $\left.q_{2}(y), \ldots, q_{T(t)}(y)\right)$. We consider two tapes $z, z^{\prime}$ in $W(t)$ such that

$$
\begin{aligned}
& \text { (i) } z\left[(1,1, \ldots, 1),\left(2^{t-1}, 2^{t-1}, \ldots, 2^{t-1}\right)\right] \\
& =x\left[(1,1, \ldots, 1),\left(2^{t-1}, 2^{t-1}, \ldots, 2^{t-1}\right)\right] \text { and } \\
& z^{\prime}\left[(1,1, \ldots, 1),\left(2^{t-1}, 2^{t-1}, \ldots, 2^{t-1}\right)\right] \\
& =y\left[(1,1, \ldots, 1),\left(2^{t-1}, 2^{t-1}, \ldots, 2^{t-1}\right)\right],
\end{aligned}
$$

(ii) the part of $z$ except for $z\left[(1,1, \ldots, 1),\left(2^{t-1}, 2^{t-1}, \ldots\right.\right.$, $\left.\left.2^{t-1}\right)\right]$ is identical with the part $z^{\prime}$ except for $z^{\prime}[(1,1, \ldots, 1)$, $\left.\left(2^{t-1}, 2^{t-1}, \ldots, 2^{t-1}\right)\right]$, and

(iii) $z\left[(1,1, \ldots, 1),\left(2^{t}, 2^{t}, \ldots, 1\right)\right]=z\left[\left(1,1, \ldots, 2^{t}\right),\left(2^{t}\right.\right.$, $\left.\left.2^{t}, \ldots, 2^{t}\right)\right]$.

By assumption, the root cell of $B$ enters the same states until time $T(t)$, for the tapes $z$ and $z^{\prime}$. Since $B$ operate in time $T(t)$ and $z$ is in $T_{2}$, it follows that $z^{\prime}$ is also accepted by $B$. This contradicts the fact that $z^{\prime}$ is not in $T_{2}$.

Let $s(t)$ be the number of different sequences of states which $c$ enters until time $T(t)$. Clearly, $s(t) \leq k^{T(t)}$. On the other hand (for any set $S$, let $|S|$ denote the number of elements of $S$.), $\left|W^{\prime}(t)\right|=2^{2^{(t-1)^{2}}}$. Since $\lim _{n \rightarrow \infty} T(t) / 2^{2^{t}}=0$ (by assumption of the lemma), it follows that $\left|W^{\prime}(t)\right|>s(t)$ for lange $t$. Therefore, it follows that for large $t$ there must exist two different tapes $x$, $y$ in $W(t)$ such that

(i) both $x\left[(1,1, \ldots, 1),\left(2^{t-1}, 2^{t-1}, \ldots, 2^{t-1}\right)\right]$

and $y\left[(1,1, \ldots, 1),\left(2^{t-1}, 2^{t-1}, \ldots, 2^{t-1}\right)\right]$

and in $W^{\prime}(t)$,

(ii) $x\left[(1,1, \ldots, 1),\left(2^{t-1}, 2^{t-1}, \ldots, 2^{t-1}\right)\right]$

$\neq y\left[(1,1, \ldots, 1),\left(2^{t-1}, 2^{t-1}, \ldots, 2^{t-1}\right)\right]$, and

(iii) $\left(q_{1}(x), q_{2}(x), \ldots, q_{T(t)}(x)\right)=\left(q_{1}(y), q_{2}(y), \ldots, q_{T(t)}(y)\right)$.

This contradicts the above Proposition 3.1, and thus the Part (2) of the lemma holds.
From Lemmas 3.1 and 3.2, we can get the following theorem.

Theorem 3.1. Let $T(t)$ be a time function such that $\lim _{n \rightarrow \infty}$ $\left[T(t) / 2^{2^{t}}\right]=0$ and $T(t) \geq t(t \geq 1)$. Then $\varepsilon[n-D F A]$ is incomparable with $£[n-D U P C A(T(t))]$.

Corollary 3.1. $£[n-D F A]$ is incomparable with $£[n-$ DUPCA $(t)]$, which is the class of sets accepted by n-DUPCA's operating in real time.

Corollary 3.2. $£[n-D F A]$ is incomparable with $£[n$ $N U P C A(t)]$.

\section{Conclusion}

In this paper, we dealt with the accepting powers of bottom-up pyramid cellular acceptors with $n$-dimensional layers, and showed that the class of sets accepted by $n-D F A$ 's is incomparable with the class of sets accepted by $n$-DUPCA's which operate in time of order lower than the diameter of the input. It is still unknown whether the class of sets accepted by $n$-DUPCA's includes the class of sets accepted by $n$-DFA's.

\section{References}

1. M.Blum and C.Hewitt, "Automata on a two-dimensional tape", in IEEE Symposium on Switching and Automata Theory (1967), pp. 155-160, 1967.

2. C.R.Dyer and A.Rosenfeld, Cellular pyramids for image analysis, Technical Report TR-544, Computer Science Center, University of Maryland, 1977.

3. K.Inoue, and A.Nakamura, Some properties of two-dimensional on-line tesselation acceptors, Information Sciences, Vol.13, pp.95-121, 1977.

4. K.Inoue, I.Takanami, and A.Nakamura, A note on two-dimensional finite automata, Information Procesing Letters, Vol.7, No.1, p.49, 1978.

5. K.Inoue, I.Takanami, Remarks on two-dimiensional finite automata with multiplication and bottom-up pyramid acceptors, Technical Report IECE of Japan, No.AL77-61, 1978.

6. K.Inoue and I.Takanami, A note on bottom-up pyramid acceptors, Information Processing Letters Vol.8, No.1, pp.34-37, 1979.

7. M.Sakamoto, S.Nogami, K.Inoue, and M.Kono, A relationships between the accepting powers of three-dimensional finite automata and time-bounded bottom-up pyramid cellular acceptors with 
three-dimensional layers, Trans. of SCI(Japan), Vol.17, No.10, pp-451-458, 2004.

8. H.Taniguchi, K,Inoue and I.Takanami, A note on three-dimensional finite automata, Information Sciences, Vol.26, pp.65-85, 1982.

9. Y.Uchida, T.Ito, H.Okabe, M.Sakamoto, H.Furutani, and M.Kono, four-dimensional multi-iinkdot finite automata, WSEAS Trans. on Computers, Issue 9, Vol.7, pp.1437-1446, 2008. 